\title{
Gestión de la compensación profesional y desempeño laboral en unidades administrativas de salud, distrito de Tarapoto, 2015 - 2016
}

Mg. Montilla Pérez, Lindsay

ORCID:0000-0002-7474-7831 lmontilla@ucv.edu.pe

Universidad César Vallejo-Tarapoto - Perú

Dr. Sánchez Dávila, Keller ORCID: 0000-0003-3911-3806 ssanchezda2081@ucvvirtual.edu.pe Universidad César Vallejo-Tarapoto - Perú

\author{
Dr. Delgado Bardales, José Manuel \\ ORCID: 0000-0001-6574-2759 \\ jmdelgadob@ucvvirtual.edu.pe \\ Universidad César Vallejo-Tarapoto - Perú \\ Scopus autor ID: 24070333700 \\ Código Renacyt: P0050554
} RESUMEN

El estudio tuvo como objetivo establecer la relación entre la compensación profesional y desempeño laboral en las áreas de administración de la Red de Salud San Martín. La investigación fue básica correlacional. Se trabajó con una muestra de 77 colaboradores; en base a ello, se aplicó la técnica de encuesta con el instrumento para recolección de datos y su posterior procesamiento y análisis. Los resultados obtenidos fueron que el nivel de compensación profesional en las áreas de administración de la Red de Salud San Martín, es "Regular" con un $45 \%$, en tanto el desempeño laboral es de $43 \%$ catalogado en el nivel "Regular". Asimismo, existe un coeficiente de correlación lineal de Pearson $r=0,923$ indicando la existencia de una relación significativa positiva entre la dimensión compensación económica con el desempeño laboral. De la misma manera existe relación significativa positiva entre la dimensión compensación No económica con el desempeño laboral con un coeficiente de correlación lineal de Pearson $r=0,873$. Finalmente, se encuentra un coeficiente de correlación lineal de Pearson $r=0,919$ indicando la existencia de una alta correlación positiva entre la compensación profesional con el desempeño laboral. Además, existe un coeficiente de determinación (0.844), explicando que aproximadamente el 84\% del desempeño laboral se da por la compensación profesional.

Palabras clave: Compensación profesional, desempeño laboral, administración 


\title{
Management of professional compensation and work performance in administrative health units, district of Tarapoto, 2015 - 2016
}

\begin{abstract}
The objective of the study was to establish the relationship between professional compensation and job performance in the administration areas of the San Martín Health Network. The research was basic correlational. We worked with a sample of 77 collaborators; Based on this, the survey technique was applied with the instrument for data collection and subsequent processing and analysis. The results obtained were that the level of professional compensation in the administration areas of the San Martín Health Network is "Regular" with 45\%, while work performance is $43 \%$ cataloged at the "Regular" level. Likewise, there is a Pearson linear correlation coefficient $r=0.923$ indicating the existence of a significant positive relationship between the economic compensation dimension and job performance. In the same way, there is a significant positive relationship between the Non-economic compensation dimension with job performance with a Pearson linear correlation coefficient $r=0.873$. Finally, a Pearson linear correlation coefficient $r=0.919$ is found, indicating the existence of a high positive correlation between professional compensation and job performance. In addition, there is a coefficient of determination (0.844), explaining that approximately $84 \%$ of job performance is due to professional compensation.
\end{abstract}

Keywords: Professional compensation, job performance, management

Artículo recibido: 15 abril 2021

Aceptado para publicación: 19 abril 2021

Correspondencia:1montilla@ucv.edu.pe

Conflictos de Interés: Ninguna que declarar 


\section{INTRODUCCIÓN}

En la actualidad el sector público viene implementando diferentes estrategias para el mejoramiento de la calidad, con aspectos tanto internos como externos, siendo en primera instancia la compensación que genera la institución en bien de los trabajadores; el mismo que está alineada con la estrategia de la organización. Un sistema de compensación está orientado a lograr trabajadores satisfechos, retener buenos talentos, resultados mejores para la empresa $u$ institución, motiva los cambios en los comportamientos de las personas que conforman el equipo de trabajo. Para tener un sistema de compensación adecuado el gerente inserta estrategias definidas, conociendo los medios y fines, para obtener los resultados esperados y al mismo tiempo conocer el recurso humano que cuenta la institución, siendo necesario identificar las métricas asociadas a las personas, más no a los cargos, que son críticas para producir ciertos resultados, no se necesita compensar a todos los empleados, basta con sólo compensar a los empleados claves que hacen que el resultado sea el esperado y detrás de esos elementos hay que garantizar que ese sistema de compensación sirva para mejorar el comportamiento de las personas de un periodo a otro, que las personas puedan aprender a mejorar su desempeño en periodos futuros. La administración de la compensación intenta no solo obtener el equilibrio interno de salarios en la organización, sino también obtener el equilibrio externo de salarios con relación al mercado de trabajo (Morales y Valencia, 2009). En base a lo indicado llegará el momento donde el salario se vuelve emocional, formando parte del paquete anual de todo trabajador del conocimiento, se convierte en una deuda, una responsabilidad que no puede ser obviada, necesaria y obligatoriamente debe formar parte de la compensación total de todo trabajador del conocimiento y por ende éste estará al pendiente de obtenerlo es allí donde el desempeño juega un papel importante dentro de las organizaciones públicas o privadas.

Por otro lado, concebir el desempeño de las personas como el activo más relevante que debe administrar la jefatura muestra que el rol de las compensaciones es direccionar el desempeño de las personas a lo que la empresa o institución espera. Esto le atribuye un carácter individual y de particularidad a las compensaciones, pues deben considerar el tipo de trabajo de la persona, los resultados que se esperan de su cargo y cómo recompensarle por los logros demostrados, tanto a nivel de remuneración como de recompensas no monetarias, es importante primero saber que los asuntos vinculados a la motivación son relevantes prácticamente todas las áreas de la psicología contemporánea, incluyendo la fisiología, la psicología social, la psicología laboral, la personalidad, el aprendizaje, la cognición, la educación, la psicología clínica y la psicología 
de la salud, el poder y el empoderamiento, sin olvidar la responsabilidad ética que tiene la empresa de otorgar el salario emocional (equilibrio emocional, bienestar físico y psicológico, en síntesis, calidad de vida) a las personas que en ella trabajan. En segundo lugar y no por eso menos importante, está el correcto manejo administrativo del caso, la correcta definición de cargos y funciones, la correcta definición de la estructura organizativa alineada a la planificación estratégica, el clima psicológico, el clima organizacional, la formación de equipos, el manejo de conflictos, la cultura, la gerencia, el liderazgo, la comunicación, las interrelaciones organizacionales y personales, la capacitación, la motivación.

El régimen laboral en el sector público contempla una serie de normas jurídicas o leyes aplicables de acuerdo a cada una de las instituciones públicas enumeradas tanto en la Constitución Política del Perú. La evaluación es un proceso para medir el rendimiento laboral del trabajador, con el objeto de llegar a la toma de decisiones objetivas sobre los recursos humanos, siendo estos fundamentales para verificar cómo se desarrollan cada actividad en el puesto de trabajo. En la Red de Salud San Martín en la ciudad Tarapoto hay personal administrativo que consideran que no son muy bien compensados por las actividades que desarrollan, el mismo que genera descontentos en cuantos a las remuneraciones. Unido a ello existes puestos laborales que satisfacen las expectativas del personal, conllevando a un disgusto, todo ello pues genera en desempeño inadecuado del personal a pesar de contar con capacidades y habilidades, por ello la investigación se planteó planteo como pregunta: ¿Cuál es la relación entre la compensación profesional y el desempeño laboral en las áreas de administración, de la Red De Salud San Martín en la ciudad Tarapoto, 2015 - 2016 ?

Entre los diferentes estudios relacionados al tema de investigación, tenemos a: Corzo (2009), Guatemala, concluye, que el sistema de compensación de la Empresa de Servicios Financieros, S.A. carece de un enfoque que verdaderamente haga valer a las compensaciones como motivador y facilitador, permitiendo impulsar a cada trabajador y a la empresa misma a ser más productiva, ya que su sistema de compensación está únicamente ligado a cuotas fijas salariales que no estimulan a los trabajadores a obtener resultados por encima de los estándares normales de desempeño; asimismo menciona también que los procesos de compensación salarial no se ha desarrollado adecuadamente, pues los colaboradores consideran que la cantidad percibida no cubre con sus necesidades básicas, ni corresponde al nivel de trabajo realizado diariamente por el personal. Además, Castro y Sajona (2012), Cartagena, concluye que los factores o pasos para el diseño de la estructura salarial solo son aplicados en el análisis y descripción de puestos, 
y la valuación de puestos, los cuales de una forma estructurada y técnica brindan la oportunidad de obtener información acerca de los puestos. Donde se tienen en cuenta las tareas, las responsabilidades, las condiciones de trabajo, las habilidades y destrezas, la experiencia, los conocimientos académicos y técnicos, así como los requisitos especiales o legales. Seguido de la valuación o valoración de puestos, el cual va a determinar la posición relativa de un puesto con respecto a los demás de la organización, incluyendo una comparación formal y sistemática entre ellos, a fin de determinar el valor de cada uno con relación a los otros. La política salarial de las empresas en estudio permite motivar y estimular a un grupo significativo de sus trabajadores pues se sienten a gusto con los beneficios que esta les otorga; sin embargo, existe un grupo significativo de empleados que afirma no tener conocimientos claros sobre este tema y le atribuyen el problema a la falta de información o a la calidad de la misma. Con respecto a la variable compensación salarial del sector examinado llegó a concluir que ésta es comprendida por sus empleados, generando ventajas para la compañía, ya que teniendo de ante mano estrategias que permitan retener, mantener y motivar al personal idóneo que permita un crecimiento económico y/o rentabilidad a la empresa.

También Pineda (2009). concluye que, que el 45\% del personal de los encuestados indican que no le importan a su jefe inmediato como persona y tampoco les cumple cuando les promete algo, que el salario que perciben no es gusto con otros compañeros que trabajan en la institución aunque tengan la misma labor, las mismas responsabilidades; tan solo el $34 \%$ de los encuestados se sienten orgullosos de trabajar en la institución y están conscientes de que mucha gente desearía tener el trabajo que ellos tienen; el $21 \%$ de los encuestados mostró duda o pena de contestar con sinceridad las preguntas planteadas, por temor o la inseguridad que tiene con respecto de las mismas y de su situación dentro de la institución, el 11\% de los encuestados se sienten más o menos motivados, o sea que no están muy seguros.

Igualmente, Gutiérrez (2010), concluye que, el $44.5 \%$ del potencial humano de la UPLA (docentes y administrativos) consideran que sus necesidades no están siendo satisfechas, un $18.8 \%$ perciben que sus necesidades son poco satisfechas y un $25.7 \%$ consideran que sus necesidades no satisfechas. El 55.5\% afirma que la UPLA si satisface sus necesidades. El 62.8\% del personal docente nombrado y el $55.7 \%$ del personal administrativo nombrado consideran que la UPLA si satisface sus necesidades. El personal docente contratado (55.1\%) y administrativo contratado (52.0\%) consideran que sus necesidades no están siendo satisfechas por la UPLA. En la jerarquía de insatisfacción de necesidades a nivel del potencial humano de 
la UPLA, los mayores niveles de insatisfacción de necesidades específicas están referidas a: Lo que ganan no es suficiente para atender a los que dependen de ellos (87.6\%). Escasos incentivos de la UPLA (comisiones, felicitaciones y otros) (82.4\%). Las remuneraciones, bonificaciones y otros que perciben no satisfacen sus necesidades básicas (80.3\%). Inseguridad e inestabilidad laboral (74.5\%). Escaso reconocimiento de la UPLA por desempeño (73.3\%). La UPLA no proporciona oportunidades de crecimiento económico (72.1\%). Escaso reconocimiento de la UPLA por la función (64.0\%). Trabajar en la UPLA no permite tener vacaciones anuales $(63.8 \%)$. No se recibe trato justo en el trabajo (54.9\%). El trabajo en la UPLA no da jerarquía ni reconocimiento social (51.7\%). Los mayores niveles de satisfacción de necesidades del potencial humano de la UPLA tienen la jerarquía siguiente: Ánimos y energías para realizar adecuadamente el trabajo (90.2 \%). Buena elección de mi profesión (89.0\%). Trabajos que desempeño acorde a mis capacidades $(87.9 \%)$. Trabajo actual muy interesante $(87.7 \%)$. Reconocimiento por la familia y amigos por la labor que realizo $(84.7 \%)$. Escasos incentivos de la UPLA (comisiones, felicitaciones y otros) (82.4\%). Buenas relaciones laborales con mi jefe $(74.8 \%)$. Sueldo, aguinaldos y vacaciones de acuerdos a ley $(73.4 \%)$. No deseo otro empleo en lugar del empleo que tengo en la UPLA (69.4\%). Estatus y jerarquías como factor muy importante en la UPLA (68.4\%). Asignación de debidas funciones y obligaciones (55.7 $\%)$. En cuanto a necesidades fisiológicas Los niveles de satisfacción e insatisfacción de necesidades fisiológicas del personal docente y administrativo se muestran a continuación: el $53.1 \%$ de los docentes nombrados y el $46.2 \%$ de los administrativos nombrados presentan los más altos índices de satisfacción de sus necesidades fisiológicas. Los índices de satisfacción de necesidades fisiológicas del personal docente contratado y del personal administrativo contratado, son sumamente bajos (34.1\% y 30.7\%, respectivamente). Las diferencias porcentuales de satisfacción de estas necesidades son muy significativas, a nivel de docentes y administrativos nombrados y contratados.

Además, Pérez (2009), concluye que, la evaluación del desempeño es una práctica extendida en el ámbito de los recursos humanos. Es un proceso en el que se intenta determinar las actitudes, rendimiento y comportamiento laboral del colaborador en el desempeño de su cargo. La evaluación del desempeño sirve para, dirigir y controlar al personal de forma más justa, y comprobar la eficacia de los procesos de selección de personal. Cuando seleccionamos a un candidato estamos haciendo una predicción sobre su rendimiento futuro y a través de la evaluación de desempeño podemos comprobar si esta predicción se ha cumplido o no. También 
nos proporciona datos sobre el clima laboral, mejorar el ajuste entre la persona y el puesto, adaptación personal al puesto, rediseño del puesto, rotación de puestos y podemos conocer las capacidades individuales, las motivaciones y expectativas de las personas para asignar los trabajos de forma adecuada. También permite detectar el potencial de los trabajadores para establecer planes de desarrollo profesional, para establecer los objetivos individuales y revisar el cumplimiento de los objetivos anteriores, así como también ver las debilidades y fortalezas de la empresa, para hacer técnicas y ejecutar planes para fortalecer esas áreas de mejora. Existen numerosos métodos y técnicas para la evaluación del desempeño laboral, en este trabajo sean mostrado lo más utilizados en las industrias, que han sido diseñados por quienes han estudiado la problemática del desempeño. El método de la escala gráfica, se refiere a la enumeración de características, del empleado en la realización de su trabajo relacionado con valores previamente establecido. Por su parte, ordena a los empleados determinando su ubicación en niveles que van del mejor hasta el peor clasificado. Por su parte el método de comparación por pares se basa en una gráfica generada por una comparación entre empleados y determinadas características definidoras del desempeño. En cuanto al método de la distribución forzada, este busca diferenciar a los empleados para detectar claramente a los sobresalientes y detectar el desempeño deficiente o inferior al promedio. El método de incidente críticos, la forma narrativa y las escalas de estimación, permiten al supervisor inmediato describir y registrar hechos positivos o negativos, del desempeño de cada subordinado.

También, Iturralde (2011), concluye que, el trabajo de campo nos ubica en un tiempo y en un espacio real, sale a flote muchas circunstancias en los distintos ámbitos, niveles, secciones que involucran al gran recurso de la cooperativa como es el Recurso Humano, hoy acertadamente llamado Capital Intelectual. El ambiente laboral de la institución se encuentra en un margen de apreciación de los trabajadores como bueno. Los empleados de la cooperativa no conocen en su mayoría los resultados de la su evaluación del desempeño. Los objetivos de la realización de la evaluación del desempeño no son conocidos por parte de los trabajadores de la Cooperativa OSCUS. No existe un plan de Mejora en función del desempeño de los trabajadores, lo que impide su desarrollo y crecimiento. No se identifican métodos, técnicas apropiadas de evaluación del desempeño que potencialicen y fortalezcan el sistema de recursos humanos a fin de que el personal se motive y se comprometa con la filosofía de la cooperativa, se continuará cometiendo errores que pueden causar graves perjuicios a la institución. 
Asimismo, Latorre (2012), concluye que, teniendo en cuenta las limitaciones que se presentan en la investigación, existiendo aportaciones en el transcurso del desarrollo las cuales son: Las prácticas de RRHH orientadas al compromiso y basadas en la aproximación "soft" está relacionada de manera positiva con el desempeño de los trabajadores tomando en cuenta las expectativas y percepciones de los empleados. Las prácticas analizadas muestran una visión positiva en los objetivos estratégicos de la empresa, siempre y cuando estén fuertemente implantadas y sean visibles para los empleados, siendo informados o no directivos de recursos humanos de la empresa. Por su parte Ortiz (2013), Huánuco - Perú. Concluye que, el conjunto de planes, métodos y elementos de Control Interno no son evaluados; a falta del manual de procedimientos y guías por cada área. En tanto el presente estudio realizado por ORTIZ muestra una variedad de conceptos importantes acerca de la variable control interno, como son la importancia dentro de la organización, métodos y elementos de aplicación, y los beneficios que esta acarrea tras su adecuada implementación y brinda indicios de cómo aplicar las distintas herramientas en una institución estatal.

El termino compensación, es uno de los más usados hoy en día para definir el salario e incentivos brindados por las empresas a sus colaboradores a fin de retenerlos, satisfacerlos e incrementar su productividad, sin embargo, este término abarca un contexto mucho más amplio e influye sobre muchos aspectos vitales para la institución. La compensación es el total de los pagos que se proporcionan a los empleados a cambio de sus servicios. Los propósitos generales de proporcionar una compensación son atraer, retener y motivar a los empleados. (Mondy, Wayne y Noe; 2009, p 326). La compensación tiene que estar alineada con la estrategia de la compañía, ya que un sistema de compensación está orientado a lograr empleados satisfechos, retener buenos talentos, resultados mejores para la empresa, motivar los cambios en los comportamientos de las personas que conforman el equipo de trabajo, es así que el elemento clave sobre cualquier sistema de compensación tiene que ver con la creación de valor para la empresa, es decir, cómo la empresa es capaz de posicionarse dentro de un mercado en el que compite, eso implica clientes satisfechos y más clientes dentro de ese mercado. (Dessler; 2011, p. 84).

La compensación (sueldos, salarios, prestaciones) es la gratificación que los empleados reciben a cambio de su labor, es el elemento que permite, a la empresa, atraer y retener los recursos humanos que necesita, y al empleado, satisfacer sus necesidades materiales, de seguridad y de ego o estatus". Según Chiavenato (2010) refiere que "la remuneración o salario es el pago total 
de dinero que se le entrega a un empleado por el cargo que ejerce y los servicios que presta por un determinado tiempo"; asimismo menciona que este factor se encuentra formada por tres componentes: primero la remuneración básica que es el pago fijo que recibe el empleado de manera regular, salario mensual o salario por hora. Segundo los incentivos salariales, donde los programas diseñados para recompensar empleados de buen desempeño, bonos, participaciones en los resultados, finalmente los beneficios que es la remuneración indirecta y se conceden a través de programas como vacaciones, transporte subsidiado, seguro de vida, seguro de salud, comedor subsidiado. Los métodos de remuneraciones también son clasificados en: remuneraciones fijas las cuales consisten en salarios homogéneos y estándar que son controlados por un órgano centralizador de administración salarial, dichas remuneraciones no incentivan ni motivan al empleado y en remuneraciones variables las cuales son flexibles y consiste fundamentalmente en remunerar a los empleados por los resultados alcanzados. (Chiavenato; 2009, p. 273). Las tendencias relacionadas con el puesto de trabajo y remuneraciones, hoy más que nunca las empresas están interesadas en no realizar cambios bruscos de personal; por el contrario, quieren mantener a sus empleados actuales; por este motivo utilizan diferentes políticas de retención, con tendencia a mejorar el salario a aquellos trabajadores capacitados, por tanto la responsabilidad de mantener un puesto de trabajo ha migrado principalmente desde la entidad al trabajador (Sánchez y Calderón, 2010, p 75-79).

Gestión de la compensación profesional: Deloitte Touche Tohmatsu Limited (2015), menciona que las organizaciones son cada vez más conscientes de que la Gestión de la compensación profesional constituye una poderosa herramienta de gestión estratégica, necesaria para canalizar los esfuerzos de los empleados en una dirección congruente con los objetivos corporativos. (P.14). La administración de la compensación es una de las tareas de recursos humanos más difíciles y desafiantes para la alta gerencia porque contiene muchos elementos y produce un impacto trascendental en las metas estratégicas de una organización. Los propósitos generales de proporcionar una compensación son atraer, retener y motivar a los empleados, es por esto que Mondy, Wayne y Noé (2009) mencionan que, para llevar una adecuada gestión de las compensaciones, se debe de tener en cuenta los siguientes aspectos:

Las compensaciones económicas, según Mondy, Wayne y Noé (2009) mencionan que las compensaciones económicas (sueldos, salarios, prestaciones) son la gratificación que los empleados reciben a cambio de su labor, es el elemento que permite, a la empresa, atraer y retener los recursos humanos que necesita, y al empleado, satisfacer sus necesidades materiales, 
de seguridad y de ego o estatus; es decir, son todos aquellos pagos en metálico o en especie, con que la organización retribuye a quienes en ella trabajan, dentro de este tipo de compensaciones tenemos: (p. 56).

La compensación no económica, siguiendo a Mondy, Wayne y Noé (2009), la compensación no económica es la satisfacción que una persona recibe del puesto o del ambiente psicológico y/o físico donde el trabajo se lleva a cabo. Este aspecto de la compensación no económica incluye factores tanto psicológicos como físicos que se encuentran en el ambiente laboral de la empresa. Puesto del trabajo, entre los factores que intervienen en dicha compensación tenemos: Variedad de habilidades, Identidad de las tareas, Importancia de las tareas, autonomía y la retroalimentación. Asimismo, para la evaluación se toma en consideración los siguientes puntos en base a lo planteado por (Mondy, Wayne y Noé 2009, p.78 y 79), detallados a continuación: Ambiente laboral, según Mondy, Wayne y Noé (2009) es el indicador que cuenta aspectos como, si la empresa cuenta con políticas sólidas, empleados competentes colegas agradables, símbolos de prestigio adecuados, condiciones de trabajo, flexibilidad en el lugar de trabajo, horario de trabajo flexible, compartir el empleo, compensación flexible, trabajo a distancia, trabajo a tiempo parcial, y retiro modificado.

Remuneración base, Según CEINSA (2015) refiere que la remuneración base hace referencia al sueldo base o salario, también llamado renta fija. Se podría definir como la remuneración o pago regular que una persona recibe por sus servicios cuando está empleada por una empresa, que establece una estructura y sistema de pago equitativo a los empleados, dependiendo de sus cargos, el mercado, el desempeño a largo plazo y las competencias. (p.98).

Incentivos, siguiendo con lo planteado por CEINSA (2015) los incentivos dependen del desempeño a largo o corto plazo, las metas, los resultados y la reducción de costos, los incentivos corresponden al componente variable de la compensación total, ya que están asociados directamente con el desempeño o productividad, es decir, existe una relación directa entre lo que el empleado hace (sus resultados) y los incentivos que obtiene. El incentivo depende de los resultados y no es algo garantizado. (p.119).

Factores determinantes de la compensación, Mondy, Wayne y Noé (2009), refieren que la teoría de la compensación nunca ha sido capaz de proporcionar una respuesta completamente satisfactoria sobre el valor del servicio de una persona que desempeña un trabajo. Aunque no hay un enfoque científico disponible, las organizaciones utilizan varios factores relevantes para determinar el salario individual. En tanto Blanchard (2012) menciona la Teoría de la 
premiación, el beneficio de la CTS - en esta teoría- surge como premio por los servicios y la fidelidad del trabajador demostrada por su permanencia, laboriosidad y colaboración en la empresa. La "fidelidad" es un criterio subjetivo, en el caso peruano la CTS se entrega incluso en los casos de despido por incurrir en falta grave. Así tenemos a la Teoría de la previsión la cual se sustenta en que el beneficio de la CTS tiene como finalidad compensar las necesidades que el trabajador pueda tener una vez cesado en su puesto.

\section{Desempeño laboral}

Según Chiavenato (2010), menciona que el desempeño es el comportamiento o medios instrumentales que se pretende poner en práctica. (p. 81). Asimismo, refiere que el desempeño humano en el cargo es extremadamente situacional y varía de una persona a otra, y de situación en situación, pues depende de innumerables factores condicionantes que influyen bastantes. Cada persona evalúa la relación costo-beneficio para saber cuánto vale la pena de hacer determinado esfuerzo. A su vez el esfuerzo individual depende de las habilidades y capacidades de la persona y de su percepción del papel que debe desempeñar (p. 81). En tanto el desempeño de los colaboradores o personal que labora en una empresa, es considera como una forma de trabajo, siendo esta también de gran importancia para las organizaciones ya que de no tener el personal un buen desempeño será poco productivo para la misma, es por ello que. "El desempeño laboral es la manera como los miembros de la organización trabajan eficazmente, para alcanzar metas comunes, sujeto a las reglas básicas establecidas con anterioridad" (Ayala, 2009, p. 34)

Así mismo, la evaluación del personal es fundamental para las organizaciones, hecho que es la base principal porque es donde los trabajadores demuestran el potencial, habilidades en el desarrollo de su puesto al que está a cargo. "Una sistemática apreciación del desempeño, del potencial de desarrollo del individuo en el cargo, afirmando que toda evaluación es un proceso para estimular o juzgar el valor, la excelencia, las cualidades de alguna persona" (Chiavenato, 2009, p. 32).

Por otro lado, las evaluaciones del desempeño tienen diversos propósitos, así como también tiene diversos usos fundamentales en las organizaciones, puesto que es fundamental, debido a que trae resultados hechos que se estableció ciertos métodos de trabajo. "La evaluación de desempeño no es un fin en sí, sino un instrumento, un medio, una herramienta para mejorar los resultados del talento humano de la organización, para lograr este objetivo básico" (Gelabert, 2012, p. 10). El rendimiento laboral se encuentra relacionado con las habilidades y 
conocimientos de cada uno de los colaboradores que son utilizados en la realización de sus actividades con el fin de lograr los objetivos establecidos. "el desempeño es influenciado en gran parte por las expectativas del empleado sobre el trabajo, sus actitudes hacia los logros y su deseo de armonía" (Bittel, 2009, p. 52). Por qué evaluar el desempeño laboral: Toda persona debe recibir retroalimentación respecto de su desempeño, para saber cómo marcha en el trabajo. Sin esta retroalimentación, las personas caminan a ciegas. Principales razones para que las organizaciones se preocupen por evaluar el desempeño de sus empleados son Según (Chiavenato, 2010): Proporciona un juicio sistemático para fundamentar aumentos salariales, promociones, transferencias y, en muchas ocasiones, despido de empleados. Permite comunicar a los empleados como marchan en el trabajo, que deben cambiar en el comportamiento, en las actitudes, las habilidades o los conocimientos.

Posibilita que los subordinados conozcan lo que el jefe piensa de ellos. La evaluación es utilizada por los gerentes como base para guiar y aconsejar a los subordinados respecto de su desempeño. (p. 82). Para evaluar el desempeño laboral Alles (2015), menciona que evaluar el desempeño no debe verse, desde la perspectiva del empleado, como un momento de "rendir examen", sino como una oportunidad de expresarse y mejorar. Las empresas que lo logran, mejoran en todos los aspectos, desde el clima laboral hasta los índices que miden rotación y calidad de vida del personal, y desde ya, también optimizan el logro de los objetivos organizacionales. Según Chiavenato (2010), la evaluación de desempeño es un proceso de revisar la actividad productiva del pasado para evaluar la contribución que el trabajador hace para que se logren objetivos del sistema administrativo. (p. 81).

Dimensiones del desempeño laboral: Según Rubio (2012), el desempeño de los trabajadores tiene como objetivo conocer cómo los empleados desarrollan su trabajo, y si lo están haciendo correctamente, para la evaluación determino las siguientes dimensiones. (p. 11). Habilidades Sociales, es la conducta o comportamiento del trabajador que influye en la mejora de su empleabilidad. Así mismo esta se determina a través de los siguientes indicadores: integración del trabajador con el resto del grupo, actitud o evaluación antes de ante cualquier situación conflictiva, control en situaciones nuevas, tolerancia con las diferentes ideas expuestas, así también la expresión de sentimientos positivos y negativos adecuadamente, asume el trabajador sus errores, así mismo acepta críticas constructivas. (p. 8)

Habilidades laborales y de rendimiento, capacidad del trabajador para conseguir con éxito las metas y objetivos de su trabajo, para cumplir con dichos objetivos es necesario determinar 
ciertos indicadores: los colaboradores cumplen con las normas de la institución, es puntual, muestra interés por aprender cosas nuevas, cumple con las tareas que se les asigna, Se preocupa por conseguir los resultados esperados de su equipo, ofrece ayuda sin solicitársela, tiene iniciativa, muestra compromiso y trabajo en equipo. Habilidades de seguridad e higiene, de seguridad, capacidad del trabajador para realizar su trabajo de forma segura y conforme a las normas, para ello es importante tomar en cuenta los siguientes indicadores: el colaborador Conoce los procedimientos de la institución en materia de seguridad, Cumple con las políticas y protocolos de la institución en materia de seguridad, conoce las señales de seguridad de su puesto, cuida los equipos y herramientas asignados, mantiene ordenada y limpia la zona de trabajo, se presenta al trabajo aseado adecuadamente, viste acorde al puesto de trabajo y la institución, asiste al trabajo descansado y con buen aspecto. (p.9)

Por lo mencionado se formuló como problema general: ¿Cuál es la relación entre la compensación profesional y el desempeño laboral en las áreas de administración de la Red de Salud San Martín, 2015 - 2016?;

Como objetivo General se propuso: Establecer la relación entre la compensación profesional y desempeño laboral en las áreas de administración de la Red de Salud San Martín, 2015 - 2016; y como objetivos específicos: Identificar el nivel de compensación profesional en las áreas de administración de la Red de Salud San Martín, 2015 - 2016; Identificar el nivel de desempeño laboral en las áreas de administración de la Red de Salud San Martín, 2015 - 2016; Determinar la relación entre la dimensión compensación económica con el desempeño laboral en las áreas de administración de la Red de Salud San Martín, 2015 - 2016; Determinar la relación entre la dimensión compensación no económica con el desempeño laboral en las áreas de administración de la Red de Salud San Martín, 2015 - 2016.

\section{ESTRATEGIAS METODOLÓGICAS O MATERIALES Y MÉTODOS}

La investigación fue básica de diseño descriptivo - correlacional, (Hernández Sampieri, 2010). La población del estudio estuvo conformada por todos los trabajadores de la Red de Salud San Martín de la ciudad Tarapoto, los mismos que se encontraron laborando bajo la modalidad de CAS y Nombrados, que sumaron un total de 52 CAS y 56 nombrados haciendo un total general de 108 colaboradores. La muestra encuestada estuvo conformada por 77 colaboradores de la Red De Salud San Martín en la ciudad Tarapoto, para ello se empleó la fórmula de cálculo muestral finita. El muestreo aplicado a esta investigación, es no probabilístico, dado que se seleccionó a un conjunto de personas que se consideraron representativas del grupo al que 
pertenecen, con la finalidad de estudiar o determinar las características de los trabajadores de la red de salud. Como técnica se empleó la encuesta, las cuales estuvo conformada por las dimensiones e indicadores de cada una de las variables en estudio y como instrumentos se empleó el cuestionario de preguntas, que estuvo direccionada a evaluar el comportamiento de cada una de las variables, teniendo como participante a los trabajadores de la Red de Salud San Martín en la ciudad Tarapoto. Una vez obtenida los datos de las encuestas aplicadas se procedió a evaluar cada una de las variables, se tabulo y grafico estas en el programa Excel, así mismo se utilizó el sistema SPSS Versión 21, en el que se tabularon los resultados de las encuestas obtenido de los colaboradores, para luego utilizar la prueba de normalidad, para observar el grado de distribución de los datos, y posteriormente se calculó el coeficiente de correlación de Pearson. En el presente trabajo de investigación se aplicaron encuestas que recaudan información cualitativa que se analizó de manera cuantitativa, es por ello que la escala de evaluación es ordinal, con respuestas abiertas: Bajo, Regular y Alto.

\section{RESULTADOS Y DISCUSIÓN}

\subsection{RESULTADOS}

Tabla 1. Nivel de compensación profesional

\begin{tabular}{|c|c|c|c|}
\hline Compensación & Valor (puntaje) & $\mathbf{N}^{\circ}$ & $\%$ \\
\hline Baja & $14-32$ & 23 & $30 \%$ \\
\hline Regular & $33-51$ & 35 & $45 \%$ \\
\hline Alta & $52-70$ & 19 & $25 \%$ \\
\hline \multicolumn{2}{|c|}{ Total } & 77 & $100 \%$ \\
\hline
\end{tabular}

Fuente: Base de datos. Cuestionario sobre compensación profesional. 2015 - 2016

Tabla 2. Nivel de desempeño laboral

\begin{tabular}{lccc}
\hline Compensación & Valor (puntaje) & $\mathbf{N}^{\circ}$ & \% \\
\hline Bajo & $24-56$ & 26 & $34 \%$ \\
Regular & $56-88$ & 33 & $43 \%$ \\
Alto & $88-120$ & 18 & $23 \%$ \\
\multicolumn{2}{c}{ Total } & $\mathbf{7 7}$ & $\mathbf{1 0 0 \%}$ \\
\hline
\end{tabular}

Fuente: Base de datos. Cuestionario sobre desempeño laboral. 2015 - 2016 
Figura 1: dimensión compensación económica con el desempeño laboral.

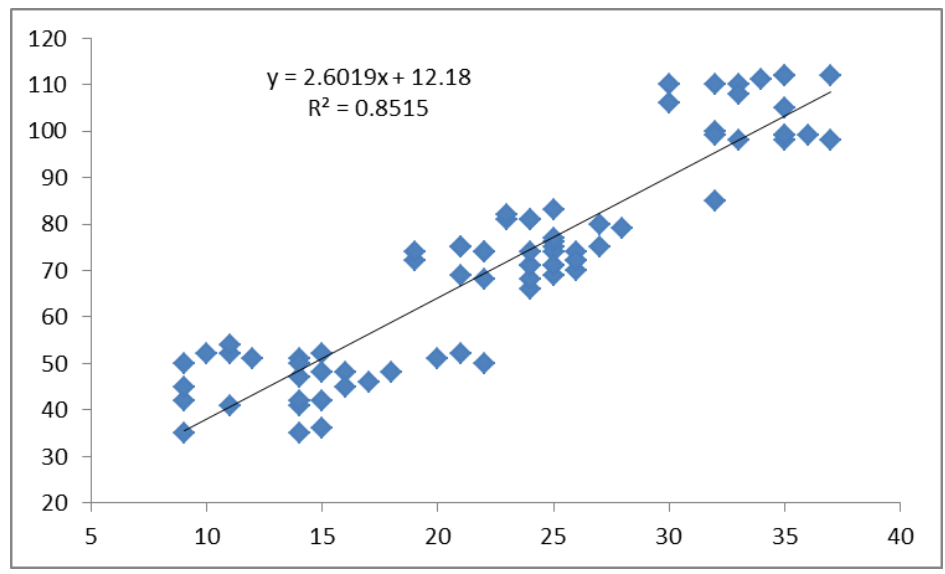

Fuente: Base de datos, Encuestas elaboradas por la autora.

Tabla 3. Resultados de la correlación de Pearson

\begin{tabular}{lc}
\hline \multicolumn{2}{c}{ Estadísticas de la regresión } \\
\hline Coeficiente de correlación & 0.923 \\
Coeficiente de determinación $\mathrm{R}^{\wedge} 2$ & 0.851 \\
$\mathrm{R}^{\wedge} 2$ ajustado & 0.849 \\
Error típico & 8.756 \\
Observaciones & 77 \\
\hline
\end{tabular}

Fuente: Base de datos, SPSS VER.21

Tabla 4. Análisis de varianza del modelo

\begin{tabular}{lccccc}
\hline & $\begin{array}{c}\text { Grados de } \\
\text { libertad }\end{array}$ & $\begin{array}{c}\text { Suma de } \\
\text { cuadrados }\end{array}$ & $\begin{array}{c}\text { Promedio } \\
\text { de los } \\
\text { cuadrados }\end{array}$ & F & $\begin{array}{c}\text { Valor } \\
\text { crítico de } \\
\text { F }\end{array}$ \\
\hline Regresión & 1 & 32954.94 & 32954.94 & 429.89 & 0.00 \\
Residuos & 75 & 5749.42 & 76.66 & & \\
Total & $\mathbf{7 6}$ & $\mathbf{3 8 7 0 4 . 3 6}$ & & & \\
\hline
\end{tabular}

Fuente: Base de datos, SPSS VER. 21 
Figura 2. Dimensión compensación no económica con el desempeño laboral.

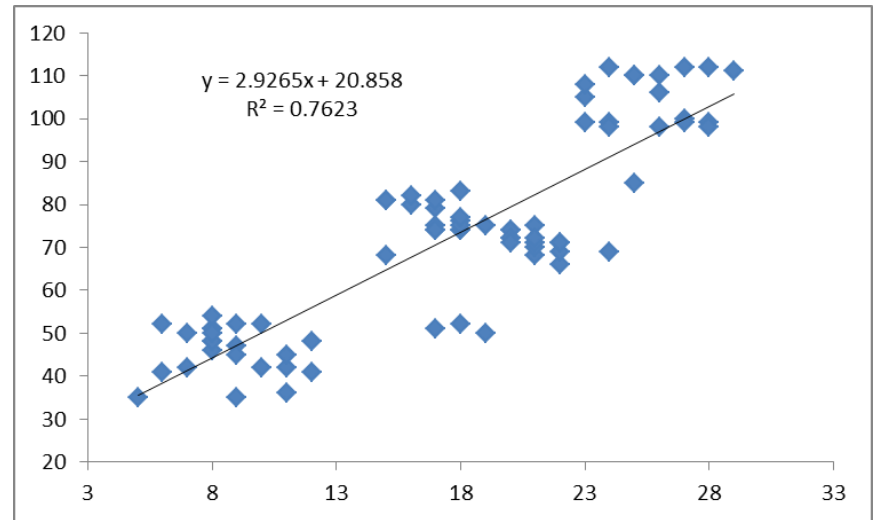

Fuente: Base de datos, Encuestas elaboradas por la autora.

Tabla 5. Resultados de La Correlación de Pearson

\begin{tabular}{lc}
\hline \multicolumn{2}{c}{ Estadísticas de la regresión } \\
\hline Coeficiente de correlación & 0.873 \\
Coeficiente de determinación $\mathrm{R}^{\wedge} 2$ & 0.762 \\
$\mathrm{R}^{\wedge} 2$ ajustado & 0.759 \\
Error típico & 11.074 \\
Observaciones & 77 \\
\hline
\end{tabular}

Fuente: Base de datos, SPSS VER. 21

Tabla 6. Análisis de varianza del modelo

\begin{tabular}{lccccc}
\hline & $\begin{array}{c}\text { Grados de } \\
\text { libertad }\end{array}$ & $\begin{array}{c}\text { Suma de } \\
\text { cuadrados }\end{array}$ & $\begin{array}{c}\text { Promedio } \\
\text { de los } \\
\text { cuadrados }\end{array}$ & F & $\begin{array}{c}\text { Valor } \\
\text { crítico de } \\
\text { F }\end{array}$ \\
\hline Regresión & 1 & 29506.127 & 29506.127 & 240.585 & 0.000 \\
Residuos & 75 & 9198.236 & 122.643 & & \\
Total & $\mathbf{7 6}$ & $\mathbf{3 8 7 0 4 . 3 6 4}$ & & & \\
\hline
\end{tabular}

Fuente: Base de datos, SPSS VER. 21

Figura 3. Ccompensación profesional con el desempeño laboral.

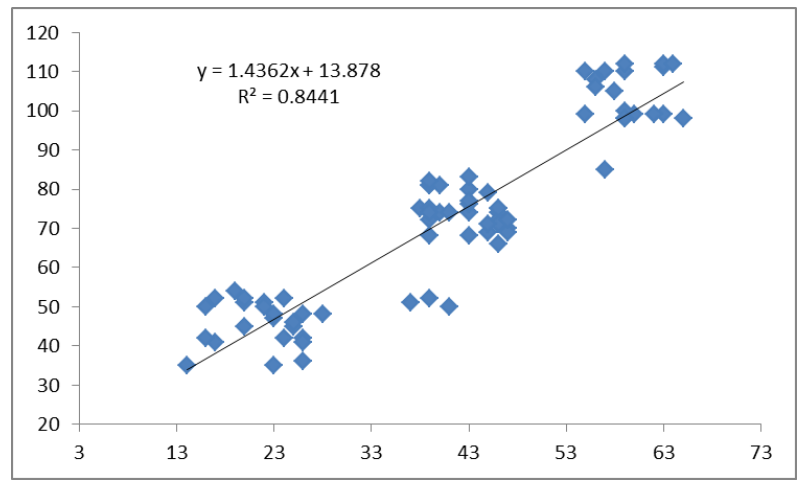


Fuente: Base de datos, Encuestas elaboradas por la autora.

Tabla 07: Resultados de La Correlación de Pearson

\begin{tabular}{lc}
\hline \multicolumn{2}{c}{ Estadísticas de la regresión } \\
\hline Coeficiente de correlación & 0.919 \\
Coeficiente de determinación $\mathrm{R}^{\wedge} 2$ & 0.844 \\
$\mathrm{R}^{\wedge} 2$ ajustado & 0.842 \\
Error típico & 8.969 \\
Observaciones & 77 \\
\hline
\end{tabular}

Fuente: Base de datos, SPSS VER. 21

Tabla 8: Análisis de varianza del modelo

\begin{tabular}{lccccc}
\hline & $\begin{array}{c}\text { Grados de } \\
\text { libertad }\end{array}$ & $\begin{array}{c}\text { Suma de } \\
\text { cuadrados }\end{array}$ & $\begin{array}{c}\text { Promedio } \\
\text { de los } \\
\text { cuadrados }\end{array}$ & F & $\begin{array}{c}\text { Valor } \\
\text { crítico de } \\
\text { F }\end{array}$ \\
\hline Regresión & 1 & 32671.15 & 32671.15 & 406.14 & 0.00 \\
Residuos & 75 & 6033.21 & 80.44 & & \\
Total & $\mathbf{7 6}$ & $\mathbf{3 8 7 0 4 . 3 6}$ & & & \\
\hline
\end{tabular}

Fuente: Base de datos, SPSS VER. 21

\subsection{DISCUSIÓN}

En el estudio de Castro y Sajona (2012) denominada "Sistemas de compensación en empresas del sector funerario de la ciudad de Cartagena", donde concluyen que los factores o pasos para el diseño de la estructura salarial solo son aplicados en el análisis y descripción de puestos, y la valuación de puestos, los cuales de una forma estructurada y técnica brindan la oportunidad de obtener información acerca de los puestos, donde se tienen en cuenta las tareas, las responsabilidades, las condiciones de trabajo, las habilidades y destrezas, la experiencia, los conocimientos académicos y técnicos, así como los requisitos especiales o legales.

Respecto a las conclusiones vertidas, el estudio muestra un coeficiente de determinación (0.844), explicando que aproximadamente el $84 \%$ del desempeño laboral se da por la compensación profesional, en tanto se puede inferir que la estructura salarial es la adecuada en funciones a los puestos y a los roles asignados en las áreas de administración de la Red de Salud San Martín.

Asimismo, Castro y Sajona (2012) refieren que la valoración de puestos, el cual va a determinar la posición relativa de un puesto con respecto a los demás de la organización, incluyendo una comparación formal y sistemática entre ellos, a fin de determinar el valor de cada uno con 
relación a los otros. La política salarial de las empresas en estudio permite motivar y estimular a un grupo significativo de sus trabajadores pues se sienten a gusto con los beneficios que esta les otorga. Según lo descrito y al relacionar la compensación profesional con el desempeño laboral en las áreas de administración de la Red de Salud San Martín, existe un coeficiente de correlación lineal de Pearson $r=0,919$ donde existe relación significativa positiva entre la compensación profesional con el desempeño laboral. Siendo este hallazgo idéntico a lo mencionado por los autores, más aún cuando mencionan que la compensación salarial genera ventajas para la compañía, permitiendo mantener y motivar al personal idóneo que facilite un crecimiento económico y/o rentabilidad a la empresa.

Por su parte Corzo (2009), en su tesis titulada "Diseño del sistema de compensación salarial para una empresa de servicios financieros", donde destaca sus principales conclusiones refiriéndose al sistema de compensación de la Empresa de Servicios Financieros, S.A. donde carece de un enfoque verdadero que haga valer a las compensaciones como motivador y facilitador, permitiendo impulsar a cada trabajador y a la empresa misma a ser más productiva, ya que su sistema de compensación está únicamente ligado a cuotas fijas salariales que no estimulan a los trabajadores a obtener resultados por encima de los estándares normales de desempeño. En tanto, el estudio desarrollado muestra que la compensación profesional en las áreas de administración de la Red de Salud San Martín es "Regular" con un 45\%; no obstante, existe un $30 \%$ de colaboradores que refieren que la compensación es "Baja"; estos resultados son similares a los mostrado Corzo quien menciona que la compensación no es impulsora para los trabajadores que coadyuven a desenvolverse de la mejor manera en la institución.

Según Iturralde (2011), en su investigación denominado evaluación del desempeño laboral y su incidencia en los resultados del rendimiento de los trabajadores de la cooperativa de ahorro y crédito OSCUS LTDA. El mismo que concluye que no existe un plan de Mejora en función del desempeño de los trabajadores, lo que impide su desarrollo y crecimiento. Asimismo, no identifican métodos, técnicas apropiadas de evaluación del desempeño que potencialicen y fortalezcan el sistema de recursos humanos a fin de que el personal se motive y se comprometa con la filosofía de la cooperativa.

En base a lo expuesto por Iturralde, el estudio muestra un nivel de desempeño laboral en las áreas de administración de la Red de Salud San Martín de "Regular" con un 43\% y solo un 23\% de colaboradores perciben que el desempeño laboral es "Alto", estos resultados aparentemente son alentadores pero no existe herramientas que permitan generar mayor desprendimiento del 
personal, siendo uno de las tantas herramientas el plan de mejora continua que debe ser abordado de manera conjunta para asegurar la participación activa de los trabajadores y con ello disminuir el $34 \%$ de desempeño "Bajo" que tiene las áreas de administración de la Red de Salud San Martín.

Para ello es necesario insertar aportes de Latorre (2012), quien menciona es su estudio titulado "La gestión de recursos humanos y el desempeño laboral”. Donde refiere que las prácticas de Recursos Humanos orientadas al compromiso y basadas en la aproximación "soft" están relacionada de manera positiva con el desempeño de los trabajadores tomando en cuenta las expectativas y percepciones de los empleados. Con ello asegurar el cumplimiento de los objetivos estratégicos de la institución, siempre y cuando estén fuertemente implantadas y sean visibles para los empleados.

Retomando a lo mencionado por Corzo (2009), en su tesis titulada "Diseño del sistema de compensación salarial para una empresa de servicios financieros", refiere también que el proceso de compensación salarial no se ha desarrollado adecuadamente, pues los colaboradores consideran que la cantidad percibida no cubre con sus necesidades básicas, ni corresponde al nivel de trabajo realizado diariamente por el personal. Sin embargo, al relacionar la dimensión compensación económica con el desempeño laboral en las áreas de administración de la Red de Salud San Martín, se encuentra un coeficiente de correlación lineal de Pearson $r=0,923$ indicando la existencia una alta correlación positiva entre la compensación económica con el desempeño laboral en las áreas de administración; inclusive muestra un coeficiente de determinación (0.851), explicando que aproximadamente el 85\% del desempeño laboral se da por la dimensión compensación económica; este resultado y aseveración que muestra el estudio es totalmente contradictorio a la conclusión que llega Corzo.

\section{CONCLUSIONES}

Al relacionar la compensación profesional con el desempeño laboral en las áreas de administración de la Red de Salud San Martín, 2015 - 2016; se encuentra un coeficiente de correlación lineal de Pearson $r=0,919$ indicando la existencia de una alta correlación positiva entre las variables; por tanto, existe relación significativa positiva entre la compensación profesional con el desempeño laboral. Además, existe un coeficiente de determinación (0.844), explicando que aproximadamente el $84 \%$ del desempeño laboral se da por la compensación profesional. 
El nivel de compensación profesional en las áreas de administración de la Red de Salud San Martín 2015 - 2016, es "Regular" con un 45\%, seguido de una compensación "Baja" con un $30 \%$ y solo un $25 \%$ de colaboradores refieren que la compensación profesional es "Alta".

El nivel de desempeño laboral en las áreas de administración de la Red de Salud San Martín, 2015 - 2016; es "Regular" con un 43\%, seguido de un desempeño "Bajo" con un 34\% y solo un $23 \%$ de colaboradores perciben que el desempeño laboral es "Alto".

Al relacionar la dimensión compensación económica con el desempeño laboral en las áreas de administración de la Red de Salud San Martín, 2015 - 2016; se encuentra un coeficiente de correlación lineal de Pearson $r=0,923$ indicando la existencia una alta correlación positiva entre las variables. Por lo tanto, existe relación significativa positiva entre la dimensión compensación económica con el desempeño laboral en las áreas de administración de la Red de Salud San Martín, 2015 - 2016. Además, existe un coeficiente de determinación (0.851), explicando que aproximadamente el $85 \%$ del desempeño laboral se da por la dimensión compensación económica.

Al relacionar la dimensión compensación no económica con el desempeño laboral en las áreas de administración de la Red de Salud San Martín, 2015 - 2016; se encuentra un coeficiente de correlación lineal de Pearson $r=0,873$ lo que indica que existe una alta correlación positiva entre las variables. Por lo tanto, existe relación significativa positiva entre la dimensión compensación No económica con el desempeño laboral en las áreas de administración de la Red de Salud San Martín. Además, existe un coeficiente de determinación (0.762), explicando que aproximadamente el $76 \%$ del desempeño laboral se da por la dimensión compensación no económica

\section{REFERENCIAS}

Alles. M, (2015), Dirección estratégica de recursos humanos. (Gestión por Competencias) 9ª edición, Buenos Aires- Argentina, ed. Granica.

Ayala, S. (2009). Proceso de Admisión y Empleo de los recursos Humanos. Lima: Ediciones San Martin.

Bittel, (2009). Desempeño laboral. Bogotá: Mc Graw Hill.

Blanchard. O. (2012), "Macroeconomía”, Madrid, editorial. Pearson.

Castro, J. y Sajona, J. (2012). Sistemas de compensación en empresas del sector funerario de la ciudad de Cartagena. Colombia: Universidad de Cartagena. 
Chiavenato, I. (2010). "Gestión del Talento Humano". 1ª edición. Bogotá, D.C.: Mc Graw Hill.

Chiavenato, I. (2009), Administración de Recursos Humanos. 10ª Edición, Colombia: Editorial Mc Graw Hill Interamericana S.A.

CEINSA (2015). “Gestión de la compensación profesional”. España: Recopilado de http://www.ceinsa.com/index.php/servicios/compensacion.html

Corzo L. (2009), Diseño del sistema de compensación salarial para una empresa de servicios financieros", Guatemala: Universidad de San Carlos De Guatemala

Deloitte T (2015). Encuesta salarial y Gestión de las compensaciones Somos su socio estratégico en la gestión de las remuneraciones y beneficios. Perú. Recopilado de http://www2.deloitte.com/pe/es/pages/humancapital/solutions/salary_survey_compensation_management.html

Dessler, G. (2011), Administración de personal, $8^{a}$ edición, México, ed. Alhambra Mexicana.

Gutiérrez, F. (2010). Motivación, desempeño y satisfacción laboral del potencial humano en la Universidad Peruana los Andes (UPLA). Perú.

Gelabert, P. (2012). Gestión de personas y el desempeño laboral en las empresas, $4^{a}$ edición, España, ed. ESIC.

Hernández, R., Fernández, C. \& Baptista, P. (2010) Metodología de la Investigación. (5ªed.). México: Mc Graw-Hill.

Iturralde, J. E. (2011) La evaluación del desempeño laboral y su incidencia en los resultados del rendimiento de los trabajadores de la cooperativa de ahorro y crédito OSCUS LTDA de la ciudad de Ambato en el año 2010.Universidad técnica de Ambato facultad de contabilidad y auditoría. Ecuador.

Latorre, (2012), "La gestión de recursos humanos y el desempeño laboral”. Universitat de Valencia - España. Servei de Publicacions

Mondy, W y Noe, R (2009), “Administración de recursos humanos”, México: editorial Pearson Educación.

Morales, J. y Velandia, N. (2010). Salarios, estrategias y sistemas salariales o de compensaciones. México: Mc Graw-Hill.

Ortiz, (2013), "Influencia Del Sistema De Control Interno Sobre El Desempeño Organizacional De La Municipalidad Provincial de Huamalí es en el Área de tesorería”, Huánuco - Perú: Universidad Privada de Huánuco. 
Pérez, A. (2009) Propuesta de un Sistema para la evaluación del desempeño laboral en una empresa Manufacturera" (tesis elaborada para obtener el grado Académico de Maestro en Ciencias en Administración). Instituto Politécnico Nacional. Ecuador

Pineda, L, (2009) Importancia del clima organizacional, y el rendimiento laboral en empleados de una Institución Pública de Administración de Justicia. Guatemala.

Rubio. F (2012), Evaluación del desempeño (herramienta practica), Andalucía - España, programa del Servicio Andaluz de Empleo para la promoción de acciones de cooperación transnacional e interregional en materia de empleo. Andalucía - España: Cooperación transnacional e interregional en el ámbito del FSE.

Sánchez, J. \& Calderón, V. (2010). Tendencias que afectan la auditoría del proceso de capacitación. Contabilidad y Auditoría (220) 